\title{
Highly Conductive Soluble Terpolymers of Aniline, Toluidine, and o-Aminobenzoic Acid/m-Aminobenzenesulfonic Acid
}

\author{
P. SAVITHA, D. N. SATHYANARAYANA
}

Department of Inorganic and Physical Chemistry, Indian Institute of Science, Bangalore, 560 012, India

\begin{abstract}
Two series of terpolymers, one of $o$ - $/ m$-toluidine and aniline with $o$-aminobenzoic acid and the other of $o$-/ $m$-toluidine and aniline with $m$-aminobenzenesulfonic acid, have been synthesized by oxidative polymerization via an emulsion method with ammonium persulfate as the oxidant and $\mathrm{HCl}$ as the external dopant. The terpolymers exhibit excellent solubility and retain the high conductivity $\left(\sim 1 \mathrm{~S} \mathrm{~cm}^{-1}\right)$ characteristic of the unsubstituted homopolymer, polyaniline. The terpolymers also possess higher thermal stability than polyaniline. This can be attributed to the presence of internal doping groups and substituents, which introduce flexibility to the otherwise rigid polyaniline backbon
\end{abstract}

Keywords: conducting polymers; emulsion polymerization; polyamines; terpolymers

\section{INTRODUCTION}

Polyaniline (PANI) has emerged as an important conducting polymer because of its good environmental, chemical, and thermal stability and ease of synthesis. PANI also has the unique ability to attain high conductivity upon doping with an acid. The doping process enhances the conductivity of PANI by almost 8 orders of magnitude. The dopants range from inorganic acids to organic acids. ${ }^{1}$ Self-doped PANI with substituents such as carboxylic, ${ }^{2}$ sulfonic, ${ }^{3}$ and phosphonic acid ${ }^{4}$ groups and the copolymers of these substituted anilines with aniline have also been synthesized and characterized. ${ }^{5-9}$ The copolymers, though generally exhibiting better solubil-

Correspondence to: D. N. Sathyanarayana (E-mail: dns@ ipc.iisc.ernet.in) ity than PANI, which is insoluble in common organic solvents, have lower conductivity.

A large number of binary copolymers of aniline and its derivatives or of two aniline derivatives have been prepared and characterized. ${ }^{10-14}$ These copolymerizations have afforded materials whose composition and properties can be controlled by the judicious choice of the comonomers and polymerization conditions. We investigated earlier binary copolymers of aniline and toluidine and of aniline and toluidine with $o$ aminobenzoic acid and $m$-aminobenzenesulfonic acid. ${ }^{15-17}$ In contrast, studies on terpolymerization involving three aniline monomers are very scanty. It should be interesting to find out how the copolymerization of two aniline monomers is influenced by the introduction of a third aniline derivative.

In this context, it was interesting to investigate terpolymers of aniline with aniline derivatives having an electron-donating group as well 
as a self-doping group because these terpolymers might possess good conductivity as well as good solubility. We report here the chemical synthesis of two series of terpolymers, namely, terpolymers of aniline, $o-/ m$-toluidine, and $o$ aminobenzoic acid and of aniline, $o-/ m$-toluidine, and $m$-aminobenzenesulfonic acid, with the aim of synthesizing terpolymers possessing good solubility and higher conductivity in their salt (doped) form. Emulsion polymerization was used for the synthesis with ammonium persulfate as the oxidant. This method helps to increase the interaction between the oxidant, dopant, and monomers because the reaction occurs in a large number of loci dispersed in a continuous phase providing a larger surface area in which the polymerization can take place. ${ }^{12}$ In this article, we demonstrate that terpolymerization not only improves the solubility but also leads to a significant increase in the conductivity over binary copolymers obtained from two aniline derivatives. This is the first report, to the best of our knowledge, on terpolymers containing electron-donating and electron-accepting/self-doping units. These terpolymers, which possess much higher conductivity $\left(\sim 1 \mathrm{~S} \mathrm{~cm}^{-1}\right)$ and better solubility (over $5 \mathrm{~g} \mathrm{~L}^{-1}$ ) in polar solvents, have been completely characterized, unlike those reported earlier.

There have been very few reports on terpolymers of aniline. Li et al. ${ }^{18}$ synthesized terpolymers of aniline, toluidine, and 2,3-xylidine, which were amorphous in the base (undoped) form and showed good solubility and high thermal stability. They also investigated terpolymers of pyridylamine, aniline, and xylidine as well as those of $m$-phenylenediamine, $o$-anisidine, and 2,3-xylidine, which were also reported to exhibit good solubility and higher thermal stability. ${ }^{19,20}$ However, the conductivity of these terpolymers has not been investigated.

\section{EXPERIMENTAL}

\section{Materials}

Aniline and $o-/ m$-toluidine (Merck) were doubledistilled before use. $o$-Aminobenzoic acid and $m$ aminobenzenesulfonic acid (Merck) and all other chemicals (AR-grade) were used as received.

\section{Measurements}

The ultraviolet-visible (UV-vis) absorption spectra of the samples were recorded at the ambient temperature for solutions prepared in dimethyl sulfoxide (DMSO) with a Hitachi U3400 spectrophotometer. Fourier transform infrared (FTIR) spectra of the samples were measured by the $\mathrm{KBr}$ pellet technique in the range of $4000-400 \mathrm{~cm}^{-1}$ with a Bruker IFS 55 instrument. Fourier transform Raman spectra were recorded with a Bruker RFS-100/S spectrometer for the powdered samples with a $\mathrm{Nd}^{3+}$-YAG laser with a laser power of $60 \mathrm{~mW}$ at the sample with $1064 \mathrm{~nm}$ for excitation. ${ }^{1} \mathrm{H}$ and ${ }^{13} \mathrm{C}$ NMR spectra were obtained with a Bruker AMX 400-MHz spectrometer with tetramethylsilane as the internal reference. Conductivity measurements were made with the four-probe technique (pressure contact) on pressed pellets obtained by the subjection of the powdered samples to a pressure of $50 \mathrm{kN} / \mathrm{m}^{2}$. The scanning electron microscopy (SEM) measurements were carried out with a JEOL JSM 5600 L scanning electron microscope. The X-ray diffraction (XRD) graphs were recorded for the powdered materials with a Siemens D5005 X-ray diffractometer with $\mathrm{Cu}$ $\mathrm{K} \alpha$ radiation. The solubility of the copolymers in their salt form was tested in the solvent DMSO.

\section{Oxidative Terpolymerization}

A typical synthesis of the terpolymer was as follows. The emulsifier (sodium lauryl sulfate; $2.25 \mathrm{~g}, 0.1 \mathrm{M}$ ) in $50 \mathrm{~mL}$ of water was added to $100 \mathrm{~mL}$ of the solvent (chloroform) under constant stirring to obtain a milky white emulsion. Equimolar $(0.033 \mathrm{M})$ mixtures of aniline, $o$-toluidine, and $o$-aminobenzoic acid were then added to it, and this was followed by the dropwise addition of $50 \mathrm{~mL}$ of the dopant, $\mathrm{HCl}$ $(1 \mathrm{M})$, and $50 \mathrm{~mL}$ of an aqueous solution of the oxidant, $0.1 \mathrm{M}$ ammonium persulfate $(5.7 \mathrm{~g})$. This mixture was stirred for $24 \mathrm{~h}$ for the reaction to continue when a greenish emulsion was obtained. The organic phase was separated and washed repeatedly with water. It was then added to $600 \mathrm{~mL}$ of acetone (nonsolvent) to precipitate the terpolymer. After $10 \mathrm{~h}$, it was filtered, washed with acetone, and dried in vacuo for $72 \mathrm{~h}$. 


\section{RESULTS AND DISCUSSION}

All the terpolymers were synthesized with equimolar quantities of the monomers. The names of the terpolymers are abbreviated on the basis of the monomers used. Thus, the terpolymer synthesized with the monomers o-toluidine (OT), aniline (A), and o-aminobenzoic acid (B) is denoted OTAB, whereas the terpolymer synthesized with $m$-toluidine (MT), aniline (A), and $o$ aminobenzoic acid (B) is denoted MTAB. Similarly, the terpolymer obtained with equimolar quantities of the monomers o-toluidine (OT), aniline (A), and $m$-aminobenzenesulfonic acid (S) is denoted OTAS, whereas MTAS denotes a terpolymer obtained with $m$-toluidine (MT), aniline (A), and $m$-aminobenzenesulfonic acid (S).

\section{Yield and Conductivity}

The yields of the terpolymers, calculated on the basis of the comonomer feed, as shown in Table 1, are moderate (ca. 46\%), except for the terpolymers of $o$-toluidine, aniline, and $m$-aminobenzenesulfonic acid, for which the yields are rather high $(84 \%)$. The electronic effects may influence the course of the formation of terpolymers, and OTAS has monomers $o$-toluidine (with an electron-donating methyl group in the ortho position) and $m$-aminobenzenesulfonic acid (with a sulfonic acid group in the highly electron-withdrawing meta position), which have favorable factors for polymerization.

The conductivities of the terpolymers are given in Table 1 . The terpolymers display rather high conductivity $(0.2-0.6 \mathrm{~S} / \mathrm{cm})$. This value is similar to that exhibited by the copolymers of aniline with $o$-aminobenzoic acid and $m$-aminobenzenesulfonic acid obtained with equimolar compositions of the comonomers but is one to two orders of magnitude higher than those of the corresponding copolymers of toluidines with $o$-aminobenzoic acid and $m$-aminobenzenesul-

Table 1. Yield, Conductivity, and Absorption Spectral Data for the Terpolymers

\begin{tabular}{ccccccccc}
\hline & $\begin{array}{c}\text { Yield } \\
\text { Terpolymer }\end{array}$ & $\begin{array}{c}\text { Conductivity } \\
(\mathrm{S} / \mathrm{cm})\end{array}$ & \multicolumn{4}{c}{$\lambda_{\text {max }}(\mathrm{nm})$} \\
\hline OTAB & 46 & 0.15 & 315 & 442 & 629 & 815 \\
MTAB & 48 & 0.37 & 315 & 449 & 615 & 862 \\
OTAS & 84 & 0.63 & 312 & 438 & 626 & 847 \\
MTAS & 45 & 0.45 & 314 & 437 & 624 & 841 \\
\hline
\end{tabular}

fonic acid. ${ }^{15,16}$ It is also two orders of magnitude higher than the conductivity exhibited by copolymers of aniline and $o-/ m$-toluidine obtained with equimolar compositions of the comonomer feed. ${ }^{17}$ The higher conductivity of the terpolymers under investigation, compared with that of aniline/toluidine copolymers, could be attributed to the presence of a self-doping acid substituent. The conductivity of the terpolymers containing aminobenzenesulfonic acid is somewhat higher than that of the terpolymers containing $o$-aminobenzoic acid. It may be related to the higher acid strength of $m$-aminobenzenesulfonic acid, in comparison with that of $o$-aminobenzoic acid, leading to a higher degree of self-doping in the terpolymers containing aminobenzenesulfonic acid.

\section{Absorption Spectra}

The absorption spectral bands of the terpolymers recorded in DMSO are listed in Table 1. The absorption spectrum of the $\mathrm{PANI} / \mathrm{HCl}$ salt shows four bands around 330, 430, 620, and $850 \mathrm{~nm},{ }^{17}$ whereas that of the homopolymer, poly(o-toluidine), occurs at $311,443,608$, and $790 \mathrm{~nm} .{ }^{12}$ The absorption peaks of the $\operatorname{poly}(m-$ toluidine $/ \mathrm{HCl}$ salt are found at $303,423,586$, and $859 \mathrm{~nm} .{ }^{12}$ The spectrum of $o$-aminobenzoic acid in DMSO exhibits two peaks at 320 and $270 \mathrm{~nm}$, and that of $m$-aminobenzenesulfonic acid shows peaks at 310 and $260 \mathrm{~nm}$.

The spectra of the terpolymers similarly consist of four major absorption bands. The first absorption band in the region of $311-316 \mathrm{~nm}$ is assigned to the $\pi-\pi^{*}$ transition of the benzenoid ring on the basis of the earlier studies. ${ }^{1,21}$ It is related to the extent of conjugation between the phenyl rings along the polymer chain. The second absorption band at 615-629 $\mathrm{nm}$ is assigned to the exciton transition from the highest occupied molecular orbital of the benzenoid to the lowest unoccupied molecular orbital of the quinoid ring. It is sensitive to the overall oxidation state of the polymer. The other two bands around 440 and $850 \mathrm{~nm}$ are assigned to the polaron transitions. ${ }^{22}$ The polaron bands are weak absorptions. The observation of polaron bands is consistent with a high degree of doping and good solubility of the conductive terpolymers in the salt form.

The absorption spectra of two terpolymers MTAB and OTAS are given in Figure 1. The spectra of the terpolymers show the band corre- 


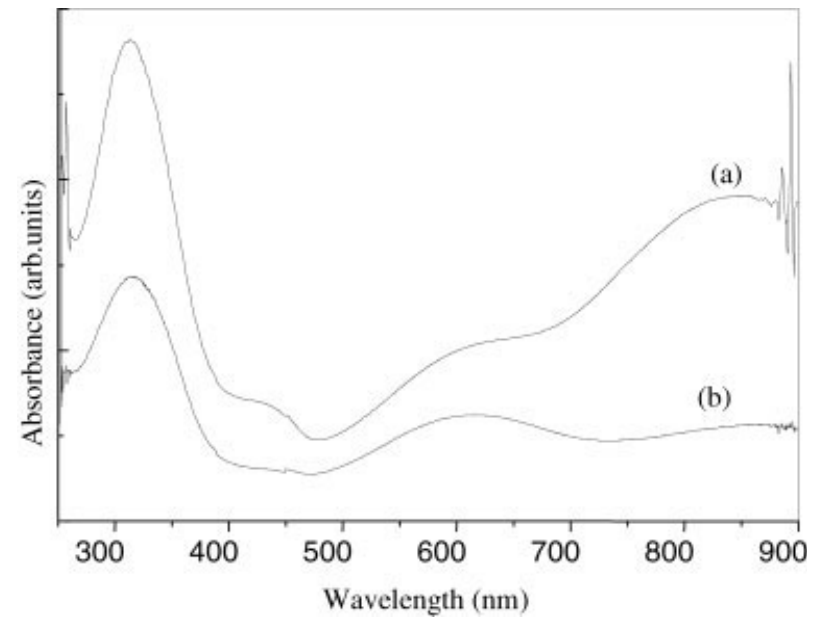

Figure 1. Absorption spectra of (a) OTAS and (b) MTAB.

sponding to $330 \mathrm{~nm}$ of PANI at $312-315 \mathrm{~nm}$. This blueshift reveals a reduction in conjugation in the terpolymers in comparison with that of PANI due to the steric hindrance caused by the ring substituents, which reduce the coplanarity of the phenyl groups along the polymer chains. In the spectra of the sulfonic acid terpolymers, the intensity of the $850-\mathrm{nm}$ peak is higher than that of the 600-nm peak. Because the former peak is associated with the formation of $-\mathrm{NH}_{2}^{+}$ species on protonation, it suggests that in the terpolymers containing $m$-aminobenzenesulfonic acid the extent of protonation is higher than those of the terpolymers containing $o$-aminobenzoic acid. This is consistent with the somewhat higher conductivity of the former terpolymers versus that of the latter terpolymers.

The position of the longest wavelength absorption peak varies according to the position of the substituents. The terpolymers having comparatively higher conductivity, namely MTAB and OTAS, show small bathochromic shifts as well as a higher intensity $850-\mathrm{nm}$ peak in comparison with those of the terpolymers OTAB and MTAS.

\section{IR Spectra}

Figure 2 compares the FTIR spectra of the homopolymer PANI with the spectrum of the terpolymer OTAS. The FTIR spectra of the terpolymers give qualitative information about the compositions of the terpolymers. The spectra of the terpolymers show the characteristic bands centered at 1600 and $1485 \mathrm{~cm}^{-1}$ corresponding to the ring-stretching vibrations of the quinoid and benzenoid rings, respectively. These peaks occur at 1559 and $1479 \mathrm{~cm}^{-1}$ in PANI and at 1587 and $1491 \mathrm{~cm}^{-1}$ in poly(o-toluidine). In the copolymers of aniline with aminobenzoic acid, these peaks are found at 1560 and $1479 \mathrm{~cm}^{-1}$, whereas for the toluidine/aminobenzoic acid copolymers, these peaks occur at 1595 and $1479 \mathrm{~cm}^{-1}$. For aniline/aminobenzenesulfonic acid copolymers, the quinoid and benzenoid peaks occur at 1560 and $1479 \mathrm{~cm}^{-1}$, and for toluidine/aminobenzenesulfonic acid copolymers, these peaks are found in similar regions. ${ }^{15,16,23}$ A medium-intensity peak can be observed at $1300-1325 \mathrm{~cm}^{-1}$ due to the $\mathrm{C}-\mathrm{N}$ stretching vibration in alternate units of the quinoid-benzenoid-quinoid rings, whereas the band at 1375-1381 $\mathrm{cm}^{-1}$ corresponds to the $\mathrm{C}=\mathrm{N}^{+}$ stretching vibration. The former band occurs at 1294 and $1315 \mathrm{~cm}^{-1}$ in the spectra of PANI and poly(o-toluidine), whereas the latter occurs at $1381 \mathrm{~cm}^{-1}$ in the spectrum of poly(o-toluidine). All the other peaks in the terpolymers are also shifted in comparison with the corresponding peaks of the homopolymers and copolymers. This shows that the polymers formed are true terpolymers and not mixtures of homopolymers and copolymers.

The $1170-1190-\mathrm{cm}^{-1}$ band in the spectra of the terpolymers can be assigned to a vibrational mode of the $\mathrm{B}-\mathrm{NH}^{+}=\mathrm{Q}$ structure, which is formed during the protonation process. It indicates the existence of positive charges in the chain and the distribution of the dihedral angle between the quinoid and benzenoid rings. It increases in intensity with an increase in the degree of doping of the polymer backbone. ${ }^{24}$ The

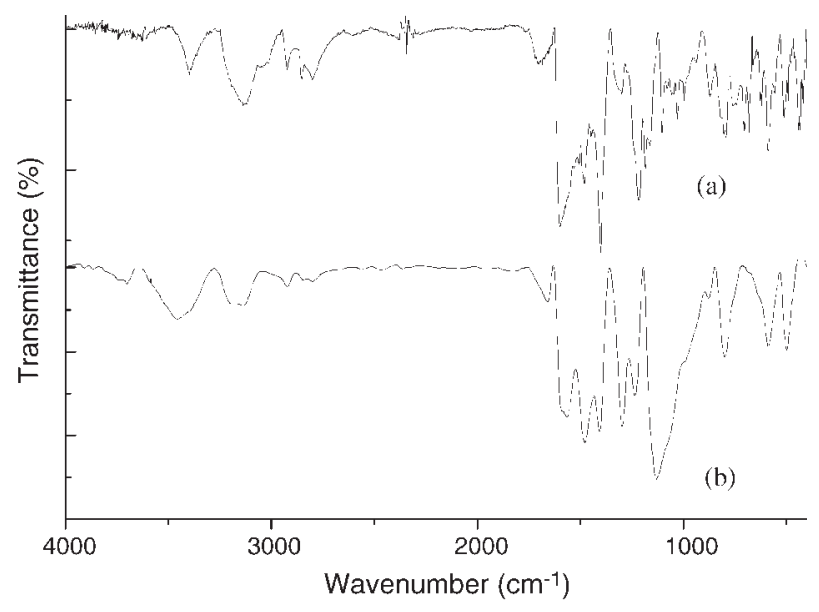

Figure 2. FTIR spectra of (a) OTAS and (b) PANI. 
bands become more intense because of the enhancement of the oscillator strengths of the backbone-related vibrations by coupling with the proton-induced charge. A band near $1107 \mathrm{~cm}^{-1}$ can be assigned to the $\mathrm{C}-\mathrm{H}$ in-plane bending. This normally IR-inactive mode becomes active when the protonation process induces conformational changes in the polymer chain, that is, by forming polarons or bipolarons. These bands confirm the presence of an aniline segment in the terpolymer backbone.

The IR spectra of the terpolymers show characteristic bands of the functional group ${ }^{25}-\mathrm{CH}_{3}$ of toluidines at about $1005 \mathrm{~cm}^{-1}$. The intense bands due to $\mathrm{C}-\mathrm{H}$ out-of-plane bending vibrations of the 1,2,4-trisubstitued benzene rings appear around 880 and $810 \mathrm{~cm}^{-1}$, and those of 1,4-disubstituted rings appear around $800 \mathrm{~cm}^{-1}$, indicating that the monomers in the terpolymers are bonded head to tail in agreement with the expected structures. The spectra of the terpolymers OTAB and MTAB display bands around 1700,760 , and $667 \mathrm{~cm}^{-1}$ arising from o-aminobenzoic acid units. The bands due to aminobenzenesulfonic acid in the IR spectra of OTAS and MTAS can be found at 1210, 1168, 1050, 695, and $620 \mathrm{~cm}^{-1}$. The bands corresponding to those of the homopolymer PANI, which occur at 1559 , $1479,1294,1238,1109$, and $801 \mathrm{~cm}^{-1}$, can be found at higher wave numbers in the spectra of the terpolymers. This indicates a lowering of the conjugation in the terpolymers possibly due to the steric hindrance of the substituents reducing the coplanarity of the phenyl rings.

The terpolymers OTAB and MTAB chiefly show bands arising from $o-/ m$-toluidine and aniline. Weak-intensity peaks of $o$-aminobenzoic acid can be found in the spectra of these two terpolymers, revealing that the extent of polymerization of $o$-aminobenzoic acid is less. On the other hand, the spectra of the terpolymers of $o / m$-toluidine, aniline, and $m$-aminobenzenesulfonic acid show more intense bands due to the sulfonic acid substituent group, revealing higher copolymerization of aminobenzenesulfonic acid units in these terpolymers. Furthermore, more intense bands due to the stretching vibrations of the sulfonic acid group can be found in the spectrum of OTAS around $1055 \mathrm{~cm}^{-1}$ in comparison with that in the spectrum of MTAS. This shows increased aminobenzenesulfonic acid units in the terpolymer OTAS in comparison with those in MTAS. Interestingly, the conductivity of OTAS is higher than that of MTAS.

\section{${ }^{1}$ H NMR Spectra}

The ${ }^{1} \mathrm{H}$ NMR spectra of the terpolymers can be discussed with reference to those of the homopolymers, PANI and polytoluidines. The ${ }^{1} \mathrm{H}$ NMR spectra of the PANI salts exhibit sharp signals near $\delta=7.3 \mathrm{ppm}$ and $\delta=6.95 \mathrm{ppm}$ due to the aromatic quinoid and benzenoid protons, respectively. A weak resonance peak at $\delta$ $=3.6 \mathrm{ppm}$ arises from the protonated $\mathrm{N}-\mathrm{H}$ protons. The ${ }^{1} \mathrm{H}$ NMR spectrum of poly(o-toluidine) salt is similarly characterized by the presence of signals in the region of $\delta=6.8-7.4 \mathrm{ppm}$ due to the protons of the aromatic rings and by a signal at $3.4 \mathrm{ppm}$ due to the $-\mathrm{NH}$ group. The additional resonances in the region of 1.8$2.1 \mathrm{ppm}$ have been assigned to the protons of the methyl group on the quinoid ring, and those in the region of 1.1-1.3 ppm have been assigned to the protons of the methyl group on the benzenoid ring. The spectrum of poly $(m$-toluidine) is very similar to that of poly(o-toluidine). The spectrum of $o$-aminobenzoic acid shows proton resonances at $\delta$ values of $6.62,6.71,7.27,7.33$, and $7.89 \mathrm{ppm}$, whereas that of aminobenzenesulfonic acid exhibits an intense singlet $(\delta$ $=7.62 \mathrm{ppm})$, a triplet $(\delta=7.47$ and $7.45 \mathrm{ppm})$, and a doublet ( $\delta=7.31$ and $7.29 \mathrm{ppm}$ ).

The ${ }^{1} \mathrm{H}$ NMR spectra of the terpolymers OTAS and MTAB are shown in Figure 3. The protons of the methyl group on the quinoid rings of toluidine can be found from 2.0 to $2.3 \mathrm{ppm}$ for the terpolymers of aniline, toluidine, and o-aminobenzoic acid, whereas they occur from 1.9 to 2.3 ppm for the terpolymers of aniline, toluidine, and aminobenzenesulfonic acid. The methyl protons on the benzenoid rings are masked by the surfactant impurities. For the terpolymers of $o$-aminobenzoic acid with $m$-toluidine and aniline, the amino protons are found at $3.7 \mathrm{ppm}$, whereas for the corresponding terpolymers with $o$-toluidine, they occur around $3.6 \mathrm{ppm}$. Aromatic protons can be found from 6.5 to $7.7 \mathrm{ppm}$ in the spectra of $o$-aminobenzoic acid terpolymers and from 6.3 to $7.6 \mathrm{ppm}$ for aminobenzenesulfonic acid terpolymers.

The aromatic protons of $o$-aminobenzoic acid undergo a small shift of $0.1-0.2 \mathrm{ppm}$ in the spectra of the terpolymers compared with that of the monomer. The peak in the region of $7.4 \mathrm{ppm}$ overlaps the resonances of PANI and poly $(o$-toluidine), which are also shifted downfield to $7.00-7.4 \mathrm{ppm}$ in the spectra of the terpolymers. The downfield shift of the aromatic protons of 


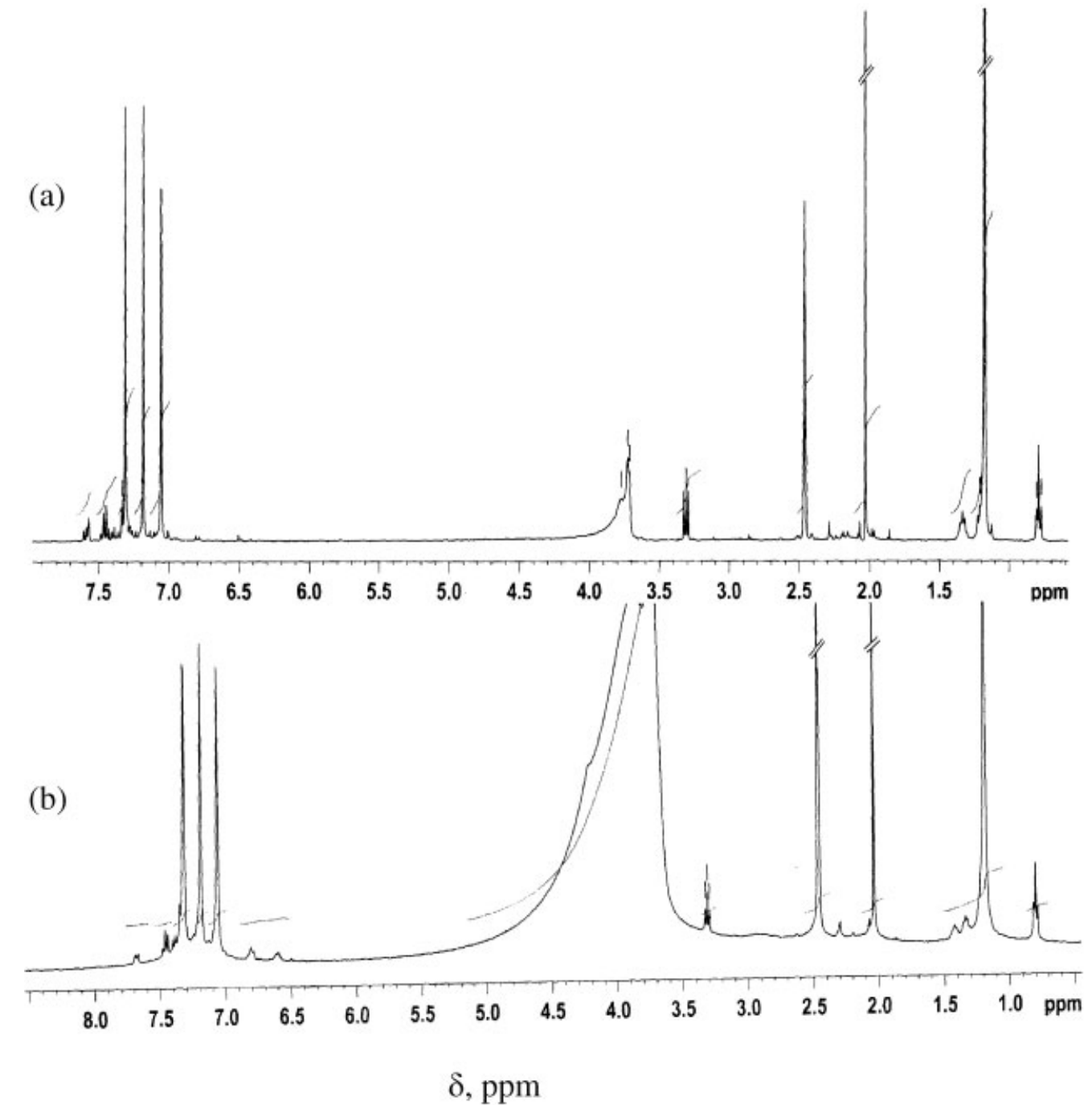

Figure 3. ${ }^{1} \mathrm{H}$ NMR spectra of (a) OTAS and (b) MTAB.

PANI and poly(o-toluidine) reveals a decrease in the electron density on their rings probably due to the presence of an electron-withdrawing carboxylic acid group of $o$-aminobenzoic acid. This conclusion is consistent with that obtained from electronic and IR spectral results.

The terpolymers containing aminobenzenesulfonic acid exhibit their aromatic proton resonances at $6.5-7.6 \mathrm{ppm}$. The singlet at $\delta=7.6 \mathrm{ppm}$ in the spectrum of aminobenzenesulfonic acid splits into a doublet and occurs around $7.57 \mathrm{ppm}$ in the spectra of the terpolymers. The triplet at $7.4 \mathrm{ppm}$ in the aminobenzenesulfonic acid monomer is observed as a multiplet at 7.4$7.5 \mathrm{ppm}$ in the spectra of the terpolymers. Furthermore, the $7.3 \mathrm{ppm}$ resonance peak of aminobenzenesulfonic acid merges with the aromatic protons of aniline and toluidine of the terpoly- mers, which occur from 7.0 to $7.3 \mathrm{ppm}$. The downfield shift of the aromatic protons could be due to the effect of aminobenzenesulfonic acid in the polymer chains decreasing the electron density of the ring.

\section{SEM}

The SEM micrographs of the terpolymers OTAS and OTAB are shown in Figure 4. The SEM pictures of the terpolymers display crystalline and amorphous morphologies. They exhibit a pattern of sharp-edged particles like the PANI salt dispersed in amorphous domains. ${ }^{26}$ The terpolymers of toluidine, aniline, and aminobenzenesulfonic acid form flakes when crushed, whereas those of toluidine, aniline, and $o$-aminobenzoic acid can easily be powdered. The SEM pictures 


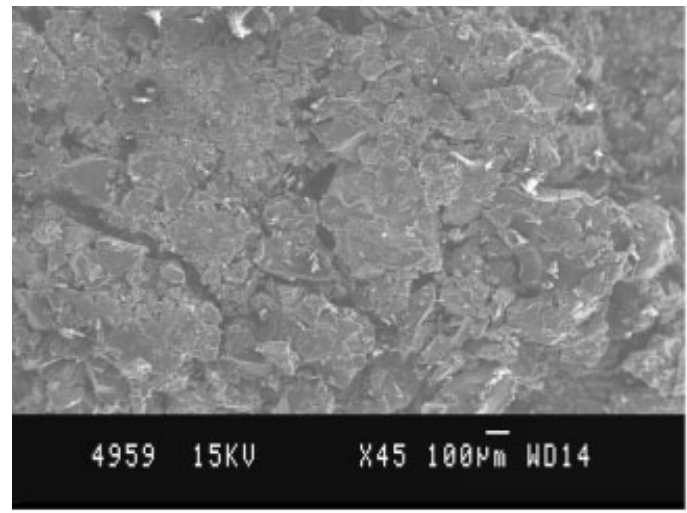

(a)

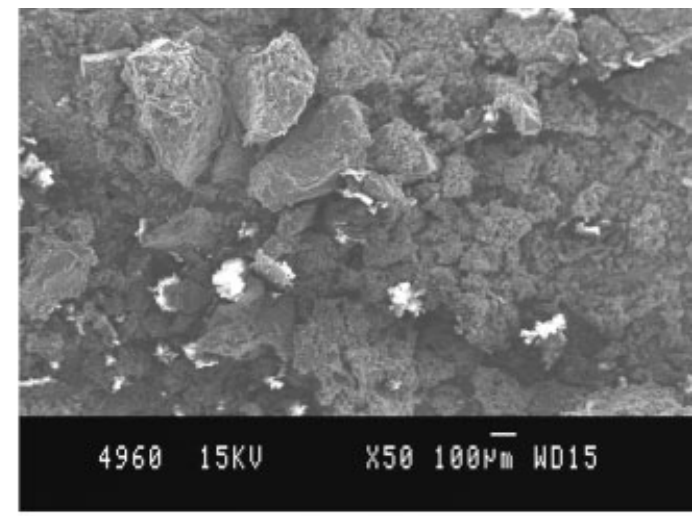

(b)

Figure 4. SEM micrographs of (a) OTAS and (b) OTAB.

of the terpolymer of $o$-toluidine, aniline, and aminobenzenesulfonic acid reveal larger particles in comparison with those of the terpolymer containing $m$-toluidine instead of $o$-toluidine. On the other hand, the terpolymers of toluidine, aniline, and $o$-aminobenzoic acid show a reverse trend: MTAB is more crystalline than OTAB. The conductivity of MTAB is also higher than that of OTAB. This is in accord with the observation that the more crystalline terpolymer generally exhibits higher conductivity than the less crystalline or amorphous terpolymer.

\section{XRD Studies}

The Bragg angles and $d$-spacings obtained from the wide-angle X-ray diffractograms of the powdered samples of the terpolymers are presented in Table 2. The number of diffraction peaks in the wide-angle $\mathrm{X}$-ray diffractograms is nearly the same for all the terpolymers. The X-ray diffractogram of the terpolymer of $m$-toluidine, aniline, and $o$-aminobenzoic acid exhibits a lower number of peaks, whereas that of the terpolymer of o-toluidine, aniline, and aminobenzenesulfonic acid contains a greater number of peaks. The terpolymers exhibit their strongest peak at $2 \theta=9-12^{\circ}$, their second strongest peak at $23-26^{\circ}$, a medium-intensity peak at $14-18^{\circ}$, and a weak peak at $28-34^{\circ}$. The X-ray powder diffractograms of the terpolymers are consistent with the results deduced from the SEM studies regarding the semicrystalline/amorphous nature of the terpolymers, with sharp peaks representing the crystalline regions found widely separated by amorphous regions with no well-defined peaks.

\section{Thermal Studies}

The thermal stability of the terpolymers was investigated with thermogravimetric analysis (TGA), differential thermal analysis (DTA), and differential thermogravimetry (DTG). TGA data are given in Table 3. Figure 5 shows the TGA and DTA curves for the terpolymer MTAS. Thermal degradation occurs in three major steps similarly to that of PANI. ${ }^{15}$ The first weightloss step, occurring between 40 and $150{ }^{\circ} \mathrm{C}$, is assigned to the loss of moisture and residual $\mathrm{HCl}$, whereas the next weight-loss step, between 180 and $320^{\circ} \mathrm{C}$, is due to the loss of dopants and side groups. The degradation of the terpolymers occurs above $420{ }^{\circ} \mathrm{C}$. All the terpolymers show

Table 2. Positions of Bragg's peaks and $d$-Spacings for the Terpolymers

\begin{tabular}{|c|c|c|c|c|c|c|}
\hline \multirow{2}{*}{$\frac{\text { Terpolymer }}{\text { OTAB }}$} & \multicolumn{6}{|c|}{$2 \theta[d(\AA)]$} \\
\hline & $9.4(9.4)$ & $11.4(7.8)$ & $13.0(6.8)$ & $24.5(3.6)$ & & $32.4(2.8)$ \\
\hline MTAB & $9.0(9.8)$ & $10.9(8.1)$ & $18.9(4.7)$ & $23.8(3.8)$ & & $32.1(2.8)$ \\
\hline OTAS & $9.3(9.5)$ & $12.1(7.3)$ & $15.4(5.7)$ & $24.7(3.6)$ & $28.4(3.1)$ & $32.9(2.7)$ \\
\hline MTAS & $9.0(9.8)$ & & $18.5(4.8)$ & $24.1(4.8)$ & & $34.1(2.4)$ \\
\hline
\end{tabular}


Table 3. TGA Data for the Terpolymers

\begin{tabular}{lcrc}
\hline & \multicolumn{3}{c}{ Temperature Range (Weight Loss) } \\
\cline { 2 - 4 } Sample & First Step & Second Step & Third Step \\
\hline OTAB & $\begin{array}{r}37-100{ }^{\circ} \mathrm{C} \\
(30 \%)\end{array}$ & $\begin{array}{r}177-283{ }^{\circ} \mathrm{C} \\
(33 \%)\end{array}$ & $440{ }^{\circ} \mathrm{C}$ \\
MTAB & $\begin{array}{r}40-150{ }^{\circ} \mathrm{C} \\
(27 \%)\end{array}$ & $\begin{array}{r}220-313{ }^{\circ} \mathrm{C} \\
(10 \%)\end{array}$ & $517{ }^{\circ} \mathrm{C}$ \\
OTAS & $\begin{array}{r}43-133{ }^{\circ} \mathrm{C} \\
(14 \%)\end{array}$ & $\begin{array}{r}230-323{ }^{\circ} \mathrm{C} \\
(16 \%)\end{array}$ & $423{ }^{\circ} \mathrm{C}$ \\
MTAS & $\begin{array}{r}39-150{ }^{\circ} \mathrm{C} \\
(29 \%)\end{array}$ & $\begin{array}{r}221-314 \\
{ }^{\circ} \mathrm{C}\end{array}$ & $534{ }^{\circ} \mathrm{C}$ \\
& \multicolumn{3}{c}{$(13 \%)$} \\
\hline
\end{tabular}

higher thermal stability in air, in the range of $420-535{ }^{\circ} \mathrm{C}$, than that of PANI, which undergoes degradation at approximately $400{ }^{\circ} \mathrm{C}$. The thermostability of the $m$-toluidine-containing terpolymers seems to be higher by $80-100{ }^{\circ} \mathrm{C}$ (degradation temperatures $=520-530{ }^{\circ} \mathrm{C}$ ) than that of the corresponding o-toluidine terpolymers (degradation temperatures $=430-440{ }^{\circ} \mathrm{C}$ ). This reveals the effect of the substituents in determining the electron densities on the ring and steric hindrances between different groups. For example, the lower thermostability of OTAS could be attributed to greater incorporation of aminobenzenesulfonic acid units in the terpolymer possibly due to lower steric hindrance of the $-\mathrm{CH}_{3}$ group of toluidine in the ortho position and of the sulfonic acid group of aminobenzenesulfonic acid in the meta position and favorable electronic effects.
The DTG curves of the terpolymers also show a three-step degradation consistent with the TG curves. The corresponding peaks in DTG occur at 82,255 , and $575{ }^{\circ} \mathrm{C}$. In the DTA curves, the first endotherm can be found around $66-82{ }^{\circ} \mathrm{C}$. The DTA peak corresponding to the second degradation step cannot be clearly seen, and the exothermic peak due to decomposition of the terpolymer occurs around $445-580{ }^{\circ} \mathrm{C}$. The terpolymer MTAS, for example, shows an endotherm at $82{ }^{\circ} \mathrm{C}$, whereas the exothermic peak due to the decomposition of the polymer occurs at $575{ }^{\circ} \mathrm{C}$.

\section{Solubility}

All terpolymers are completely soluble in polar solvents such as DMSO, dimethylformamide, and $N$-methylpyrrolidone $(\sim 5 \mathrm{~g} / \mathrm{L})$ and partially soluble in less polar solvents such as chloroform. However, the terpolymers containing sulfonic acid have the highest solubilities; OTAS is most soluble.

\section{CONCLUSIONS}

The emulsion pathway has been employed to synthesize terpolymers of $o$-toluidine $/ m$-toluidine and aniline with $o$-aminobenzoic acid $/ m$-aminobenzenesulfonic acid by chemical oxidative polymerization with ammonium persulfate as the oxidant at the ambient temperature with $\mathrm{HCl}$ as the dopant. The FTIR, UV-vis, and ${ }^{1} \mathrm{H}$ NMR

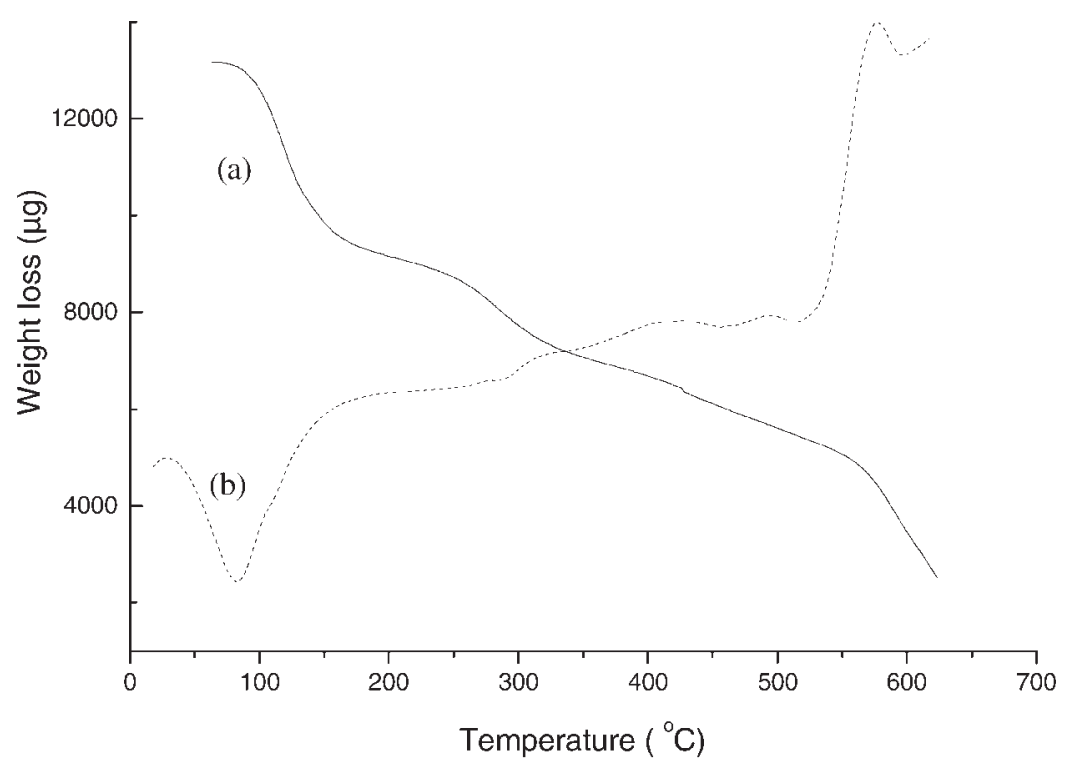

Figure 5. (a) TGA and (b) DTA curves of MTAS. 
investigations suggest that the polymers obtained are real terpolymers consisting of three monomer units. The terpolymers exhibit excellent solubility and higher thermostability than PANI. Interestingly, the terpolymers exhibit higher conductivity $(\sim 1 \mathrm{~S} / \mathrm{cm})$, although two substituted aniline segments are also present in the polymer chain.

\section{REFERENCES AND NOTES}

1. Trivedi, D. C. In Handbook of Organic Conductive Molecules and Polymers; Nalwa, H. S., Ed.; Wiley: Chichester, England, 1993; Vol. 2, p 505.

2. Toshima, N.; Yan, H.; Gotoh, Y.; Ishiwatari, M. Chem Lett 1994, 2229.

3. Karyakin, A. A.; Maltsev, I. A.; Lukachova, L. V. J Electroanal Chem 1996, 402, 217.

4. Ng, S. C.; Chan, H. S. O.; Huang, H. H.; Ho, P. K. H. J Chem Soc Chem Commun 1995, 1327.

5. Nguyen, M. T.; Diaz, A. F. Macromolecules 1995, 28, 3411.

6. Neoh, K. G.; Kang, E. T.; Tan, K. L. Synth Met 1993, 60, 13.

7. Hua, F.; Ruckenstein, E. J Polym Sci Part A: Polym Chem 2005, 43, 1090.

8. Salavagione, H. J.; Acevedo, D. F.; Miras, A. C.; Motheo, A. J.; Barbeo, C. A. J Polym Sci Part A: Polym Chem 2004, 42, 5587.

9. Fan, J.; Wan, M.; Zhu, D. J Polym Sci Part A: Polym Chem 1998, 36, 3013.
10. Rivas, B. L.; Sanchez, C. O.; Bernede, J.-C.; Basaez, L. J Appl Polym Sci 2003, 90, 706.

11. Kilmarin, P. A.; Wright, G. A. Synth Met 1997, 88, 163.

12. Savitha, P.; Sathyanarayana, D. N. J Polym Sci Part A: Polym Chem 2004, 42, 4300.

13. Li, X.-G.; Zhou, H.-J.; Huang, M.-R. J Polym Sci Part A: Polym Chem 2004, 42, 6109.

14. Fan, J.; Wan, M.; Zhu, D. J Polym Sci Part A: Polym Chem 1998, 36, 3013.

15. Rao, P. S.; Sathyanarayana, D. N. Polymer 2002, 43, 5051.

16. Rao, P. S.; Sathyanarayana, D. N. J Polym Sci Part A: Polym Chem 2002, 40, 4065.

17. Savitha, P.; Sathyanarayana, D. N. Polym Int 2004, 53, 106.

18. Li, X.-G.; Huang, M.-R.; Zhu, L.-H.; Yang, Y. J Appl Polym Sci 2001, 82, 790.

19. Li, X.-G.; Huang, M.-R.; Pan, P.; Zhu, Z.-L.; Yang, Y.-L. Polym Degrad Stab 2001, 71, 333.

20. Li, X.-G.; Duan, W.; Huang, M.-R.; Yang, Y.-L. J Polym Sci Part A: Polym Chem 2001, 39, 3989.

21. Kim, Y. H.; Foster, C.; Chiang, J.; Heeger, A. J. Synth Met 1989, 29, 285.

22. Tzou, K.; Gregory, R. V. Synth Met 1993, 53, 365.

23. Rao, P. S.; Sathyanarayana, D. N. Synth Met 2003, 138, 519.

24. Ping, Z. J Chem Soc Faraday Trans 1996, 92, 3063.

25. Silverstein, R. M.; Webster, F. X.Spectrometric Identification of Organic Compounds, 6th ed.; Wiley: New York, 1998.

26. Rao, P. S.; Sathyanarayana, D. N.; Palaniappan, S. Macromolecules 2002, 35, 4988. 